\title{
Recovery from Auditory Neuropathy Spectrum Disorder Post-Traumatic Brain Injury: A Case Study
}

\section{Suman S Penwal* and Chandrahas U Chandanshive}

Department of Audiology and Speech Therapy, Topiwala National Medical College, Mumbai, Maharashtra, India

*Corresponding Author:Suman S Penwal, Department of Audiology and Speech Therapy, Topiwala National Medical College, Mumbai, Maharashtra, India.
Received: May 04, 2021

Published: May 15, 2021

(C) All rights are reserved by Suman $\mathbf{S}$

Penwal and Chandrahas U Chandanshive.

\begin{abstract}
Background: Auditory neuropathy spectrum disorder is a retrocochlear pathology. It is marked by an abnormal transmission of stimuli by the auditory nerve. The purpose of the study is to illustrate the audiological profile of the infant with post-traumatic brain injury and subsequent recovery observed in follow-ups.

Methods: In the present case study report a 3-month-old infant was undertaken for detailed audiological evaluations and follow-up. The infant was suffering from right-sided paresis post head injury. The case underwent the diagnostic audiological test battery using standard norms and protocols. All the test procedures were non-invasive and complied with the declaration of Helsinki.

Results: The audiological test array suggested the presence of auditory neuropathy spectrum disorder in the left ear. However, the absence of contralateral reflexes in the opposite/ better ear was suggestive of bilateral retrocochlear pathology. The repeat evaluation revealed recovery from the retrocochlear pathology.

Conclusion: The recovery was attributed to the activity-dependent neuroplasticity and spontaneous drainage of neurotoxic blood accumulation in the brain.

Keywords: Auditory Neuropathy Spectrum Disorder; Recovery; Traumatic Brain Injury
\end{abstract}

\section{Introduction}

Traumatic Brain Injury (TBI) is reported to encompass a range of severity and can be caused by various stimuli as reported by Graham, McIntosh [1]. It was labeled as a "silent epidemic" and was found to affect 69 million individuals every year in Southeast Asia and Western Pacific regions [2]. Haarbauer-Krupa, Haileyesus [3] reported that TBI in children younger than 4 years of age is majorly caused by unintentional falls due to their actions (running) and the caregivers' action (carrying).

Chang, Hsu [4] demonstrated that children with TBI have an increased risk of developing speech and language disorders secondary to Attention Deficit Hyperactivity Disorder, Autism, and Developmental delay. However, Purcell, Reiss [5] evaluated 356 cases with TBI and reported that children have better resilience as opposed to adults. This can be attributed to better neuroplasticity in children. Oleksiak, Smith [6] had found that hearing dysfunction was observed in individuals post-TBI in veterans. They found an extensive variety of hearing loss among the veterans who had traumatic brain injury varying from conductive hearing loss, sensorineural hearing loss, to higher auditory deficits.

Auditory neuropathy spectrum disorder (ANSD) is a hearing disorder that impairs speech perception abilities by altering neural connections. It is found to affect the connections between the hair cells and the auditory nerve ending, or between auditory nerve synapses [7]. The prevalence of auditory neuropathy was reported to vary from $<1 \%$ to $10 \%$ as reported in the literature worldwide [8-10]. Vignesh, Jaya [8] also found that $5 \%$ of children diagnosed with sensory neural hearing loss had auditory neuropathy in India. The various causes of auditory neuropathy involve gene disorders, hyperbilirubinemia, mumps, traumatic injuries, premature delivery, hypoxia, epilepsy attacks, and ataxic motor neuropathy [7,11-13]. Few other cases reported acute ANSD post sequelae of chikungunya, post pontine hemorrhage $[14,15]$. Also, there are very few western studies that discuss early-onset ANSD [16].

The management options are highly debated in literature with few supporting the use of hearing aids $[17,18]$ and cochlear 
implants [19]. There are also studies professing complete recovery in individuals with auditory neuropathy [20,21]. From this, Psarommatis, Riga [21] inferred that the decision about the intervention for hearing in infants with ANSD that involves amplification and cochlear implant should rest till the child attains six months of age [21]. The current study aims to highlight the auditory profile of a three-months-old infant diagnosed with auditory neuropathy spectrum disorder and unilateral paralysis (Hemiparesis) and moderate to severe visual damage post-TBI with complete recovery observed in hearing abilities after six months.

\section{Case Study}

A three-month-old child was brought to the audiology clinic of the rehabilitation center in South Mumbai by parents after getting a referral from a neurologist. He was dropped from the caregiver's arm from 10 feet height at two months of age. Following the drop, the infant had right-sided hemiparesis with moderate to marked reduction in optic nerve function. Parents were not suspecting any change in hearing abilities and brought the child only because they were referred by the neurologist.

The child was taken up for detailed audiological evaluation that included behavioral observation audiometry as the child lacked neck control to perform visual reinforcement audiometry (Maico MA42, Maico Diagnostics GmbH, Germany). The child also underwent tympanometry and reflexometry (AT235, Interacoustics, Denmark); otoacoustic emission screening Distortion Product and Transient Evoked Otoacoustic Emissions (Neuro-Audio-Screen, Neurosoft, Russia); and screening for Auditory Brainstem evoked Responses (ABR) at $35 \mathrm{dBnHL}$ and 60 dBnHL was done using CE-Chirp stimulus (Neuro-Audio-Screen, Neurosoft, Russia). Additionally, the detailed diagnostic evaluation of ABR was done using Click and $500 \mathrm{~Hz}$ Tone burst stimulus (Duet, Intelligent Hearing Systems, U.S.A). Clinical neurological evaluations and brain MRI (Figure 1 and 2) was done by the neurologist and visual evoked potentials were administered by the ophthalmologist. The standard procedures were employed to carry out all the evaluations.

\section{Ethical considerations}

In the present study, non-invasive testing procedures were used. All the conditions complied with the declaration of Helsinki. All the test procedures were explained to the family members before testing and informed consent has been taken from the parents.

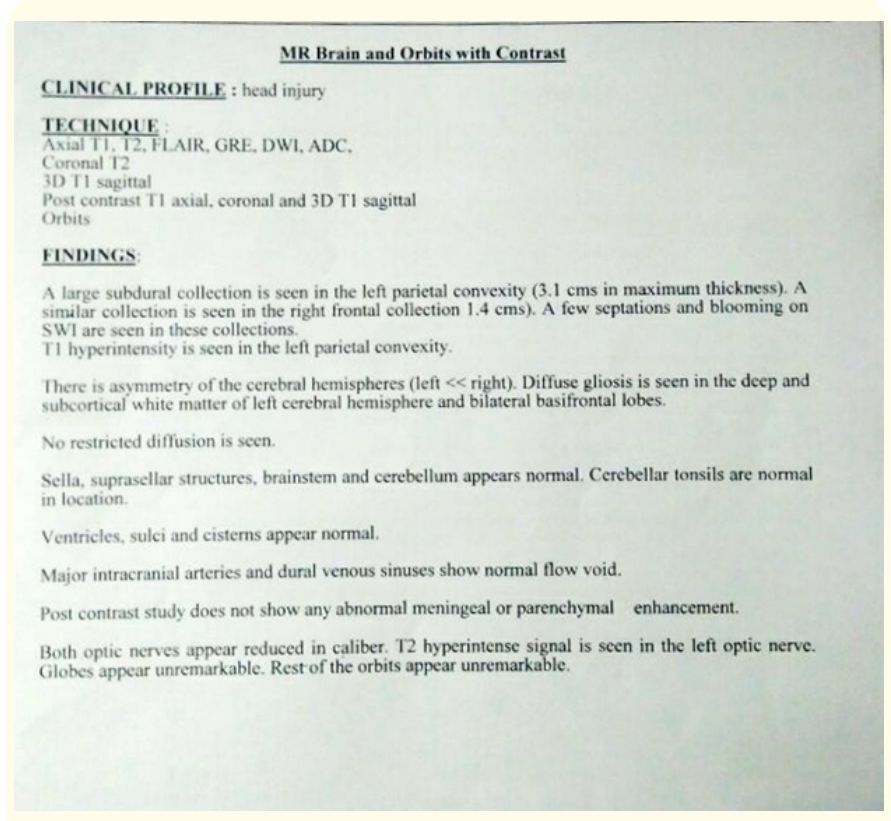

Figure 1: M.R.I report of the child with traumatic brain injury reporting diffused gliosis in the cortices (front).

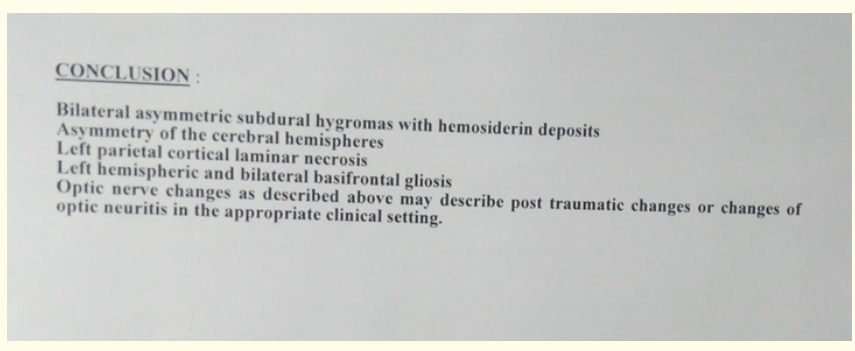

Figure 2: M.R.I report of the child with traumatic brain injury reporting diffused gliosis in the cortices (back).

\section{Results}

The data collection was done in three visits of the infant to the rehabilitation center in Mumbai and findings are given accordingly,

Visit 1: The infant was 3-month-old. He was taken up for behavioral observation audiometry. He was awake and calmly seated on the mother's lap wearing the headphones. The clinician observed response to $45 \mathrm{~dB}$ SPL in both ears for warble tone delivered through TDH-39 headphones (Telephonics, 815 Broad Hollow Road, Farmingdale, NY) housed in MX-41AR cushion. The responses observed were eye-widening and eye movements in search of the sound source. However, an inverted 'L' Jerger pattern was observed 
on acoustic reflexes for $1 \mathrm{kHz}$ tone with absent ipsilateral and contralateral reflexes in the left ear. This was observed despite the ' $\mathrm{A}$ ' type tympanogram and healthy tympanic membrane on Otoscopic evaluation in both the ears. Absent acoustic reflexes with the presence of behavioral responses to sound warranted further physiological evaluations.

The presence of otoacoustic emissions in both the ear and the absence of ABR in the left ear at both levels cleared the diagnostic picture. Also, prolonged Cochlear Microphonics was observed at moderate to higher presentation levels in the left ear ABR recordings (Figure 3), stimulus artifacts were ruled out by pitching the tube of the transducer. The child was diagnosed with unilateral auditory neuropathy secondary to TBI. Additionally, the absence of left contralateral reflexes was suggestive of mild retrocochlear pathology on the right side. The speech and language evaluations revealed a delay of 3 months in receptive and expressive language on the Receptive-Expressive Emergent Language test (REEL). The parents were counseled about regular monthly follow-up and speech and language stimulation therapy at the center.
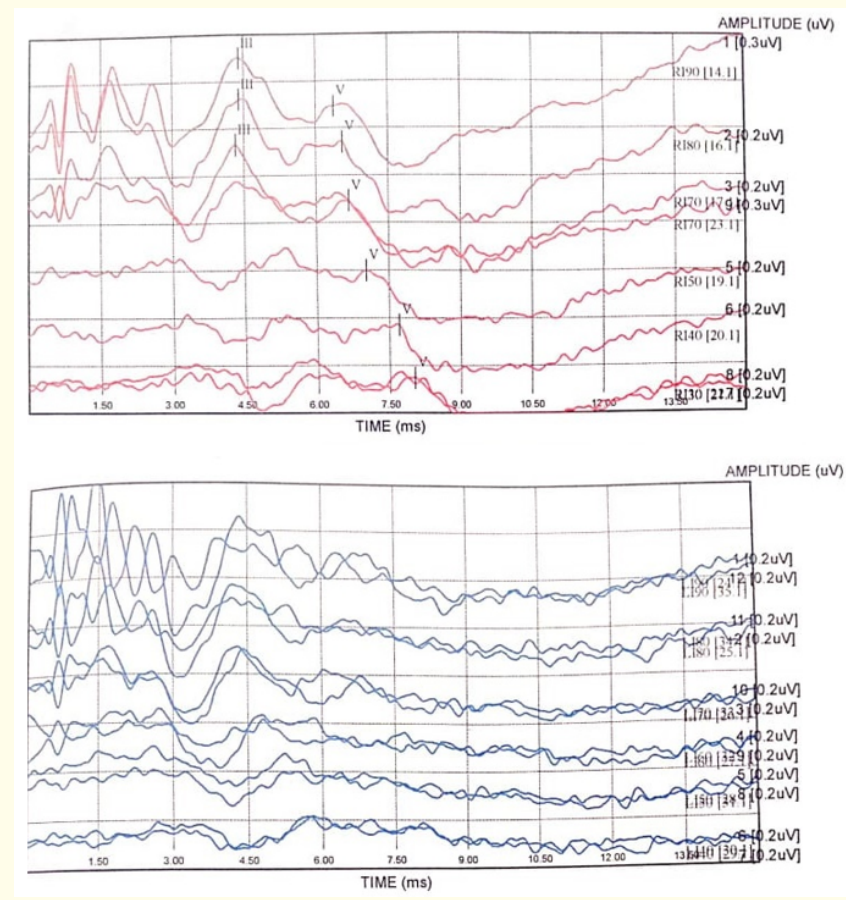

Figure 3: Waveform of Auditory evoked brainstem responses of the infant at 3 months of age.

*Note: Red $=$ Findings in right ear, Blue = Findings in left ear.
Visit 2: In the second session, after two months, it was observed that the ipsilateral reflexes were present in both ears. However, contralateral reflexes remained absent. Similarly, ABR screening revealed pass in the left ear at $60 \mathrm{~dB} \mathrm{nHL}$ and fail at $35 \mathrm{~dB} \mathrm{nHL}$. Rest all the test findings remained unchanged on the second visit. Parents were asked to continue speech therapy and follow up in the next month for audiological evaluations.

Visit 3: The child has completed seven months of age and the mother reported that the child has started to smile when spoken to and tracks the speaker. On repeating detailed audiological evaluation it was found that the entire test findings came within normal limits. Along with, diagnostic ABR evaluation demonstrated the presence of wave $\mathrm{V}$ till $10 \mathrm{~dB} \mathrm{nHL}$ in both ears (Figure 4). During these visits, the child was attending speech therapy and physiotherapy (neurodevelopmental therapy) once a week at the clinic and was on medications given by the neurologist.

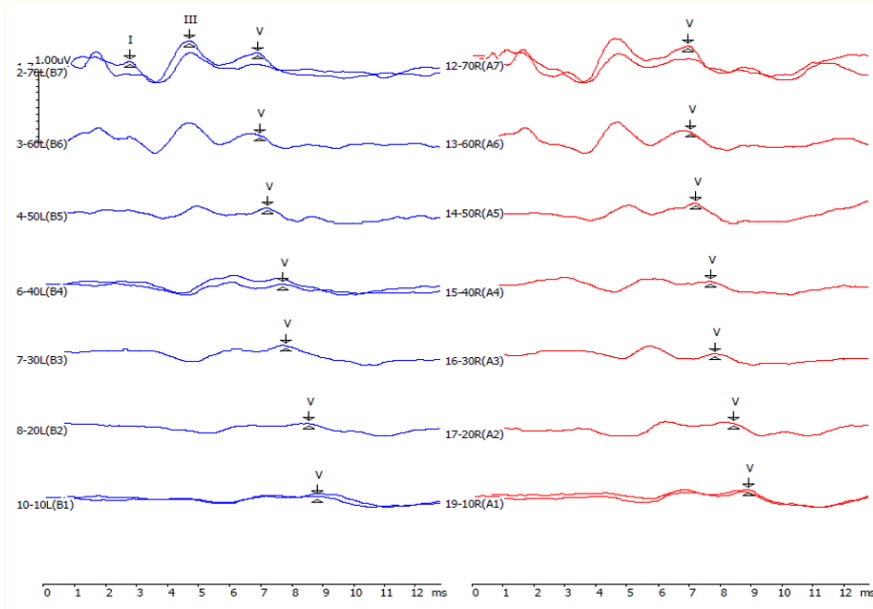

Figure 4: Waveform of Auditory evoked brainstem responses of the infant at 7 months of age.

*Note: Red $=$ Findings in right ear, Blue $=$ Findings in left ear.

\section{Discussion}

The absence of ABR in the left ear can be attributed to the bilateral diffuse cortical gliosis as reported in the MRI (Figure 1 and 2). The recovery observed in six months from the trauma can be attributed to two components; first, the activity-dependent neuroplasticity, as summarized by Ganguly and Poo [22] a specific task-oriented behavior could redistribute the cortical nerve allocation. This could be due to regular auditory input facilitated by intense language stimulation. The observation made by Scheibel, Newsome [23] 
seconds the above finding, reporting over activation of the brain to partially improve cognitive functioning. Secondly, the spontaneous drainage of neurotoxic subdural accumulation in the brain added to the recovery. Psarommatis, Riga [21] tested 177 neonates and reported spontaneous recovery of auditory neuropathy in 13 out of 20 infants on follow-up. They concluded that one should wait for six months before making any amplification decisions in the clinics based on the high-risk register and clinical findings.

Dewan, Rattani [2] found that reactive astrocytes can increase excitatory synaptic strength. Other authors have also found that reactive astrocytes maintain the homeostatic synaptic plasticity required to obtain effective brain circuit functioning. This modulation in the firing of neurons was reported to be regulated by the production of $\alpha$-amino-3-hydroxy-5-methyl-4-isoxazole propionic acid receptor and cytokine TNF $\alpha[24,25]$. It was also explained by Burda, Bernstein [26] that Astrocytes are responsible for posttraumatic tissue repair and demonstrate good potential in speeding recovery.

\section{Conclusion}

The present study reported the audiological profile of a child who acquired auditory dyssynchrony after TBI. The child also presented with marked damage in the functioning of the visual nerve and hemiparesis on the right side as diagnosed by the respective members of the medical team. The subsequent follow-up revealed complete audiological recovery from auditory dyssynchrony pattern. Hence, the current study supports the inference drawn by Psarommatis, Riga [21] to rest the intervention till the child diagnosed with ANSD, who otherwise demonstrates normal hearing unilaterally on the behavioral and electrophysiological auditory test, attains six months of age.

The parents were asked to follow up once in three months for regular audiological and speech and language developmental screening. Further research is required to support the probable cause of the recovery. The limitation of the current study is that child's hearing evaluations were not done before the traumatic injury and hence the presence of congenital auditory spectrum disorder cannot be ruled out. However, the unilateral paresis was not present since birth and was reported after the trauma.

\section{Acknowledgements}

The authors would like to thank the parents of the infant for their co-operation.

\section{Funding}

No funding sources.

\section{Conflict of Interest}

The author declares no conflict of interest in the present study.

\section{Ethical Approval}

Not required.

\section{Bibliography}

1. Graham DI., et al. "Recent advances in neurotrauma”. Journal of Neuropathology and Experimental Neurology 59 (2000): 641651.

2. Dewan MC., et al. "Estimating the global incidence of traumatic brain injury”. Journal of Neurosurgery 130.4 (2018): 10801097.

3. Haarbauer-Krupa J., et al. "Fall-related traumatic brain injury in children ages 0-4 years". Journal of Safety Research 70 (2019): 127-133.

4. Chang H-K., et al. "Traumatic brain injury in early childhood and risk of attention-deficit/hyperactivity disorder and autism spectrum disorder: a nationwide longitudinal study". The Journal of Clinical Psychiatry 79.6 (2018).

5. Purcell LN., et al. "Survival and functional outcomes at discharge after traumatic brain injury in children versus adults in resource-poor setting". World Neurosurgery (2020).

6. Oleksiak M., et al. "Audiological issues and hearing loss among Veterans with mild traumatic brain injury". Journal of Rehabilitation Research and Development 49.7 (2012).

7. Moser T and Starr A. "Auditory neuropathy-neural and synaptic mechanisms". Nature Reviews Neurology 12.3 (2016): 135.

8. Vignesh S., et al. "Prevalence and audiological characteristics of auditory neuropathy spectrum disorder in pediatric population: a retrospective study". Indian Journal of Otolaryngology and Head and Neck Surgery 68.2 (2016): 196-201.

9. Penido RC and Isaac ML. "Prevalence of auditory neuropathy spectrum disorder in an auditory health care service". Brazilian Journal of Otorhinolaryngology 79.4 (2013): 429-433.

10. Foerst A., et al. "Prevalence of auditory neuropathy/synaptopathy in a population of children with profound hearing loss". International Journal of Pediatric Otorhinolaryngology 70.8 (2006): 1415-1422. 
11. Han K-H., et al. "ATP1A3 mutations can cause progressive auditory neuropathy: a new gene of auditory synaptopathy". Scientific Reports 7.1 (2017): 1-11.

12. Ngo RY., et al. "Auditory neuropathy/auditory dys-synchrony detected by universal newborn hearing screening". International Journal of Pediatric Otorhinolaryngology 70.7 (2006): 1299-1306.

13. Narne VK., et al. "Audiological profiling of 198 individuals with auditory neuropathy spectrum disorder". Hearing, Balance and Communication 12.3 (2014): 112-120.

14. Chung S-H., et al. "A case of auditory neuropathy caused by pontine hemorrhage in an adult". Journal of Audiology and Otology 21.2 (2017): 107.

15. Prabhu P. "Acquired auditory neuropathy spectrum disorder after an attack of chikungunya: case study". European Archives of Oto-Rhino-Laryngology 273.1 (2016): 257-261.

16. Berlin CI., et al. "Multi-site diagnosis and management of 260 patients with Auditory Neuropathy/Dys-synchrony (Auditory Neuropathy Spectrum Disorder*)". International Journal of Audiology 49.1 (2010): 30-43.

17. Prabhu P and Barman A. "Effectiveness of low-cut modified amplification strategy and channel-free hearing aid in individuals with auditory neuropathy spectrum disorder". International Journal of Audiology 56.10 (2017): 759-766.

18. Walker E., et al. "Children with auditory neuropathy spectrum disorder fitted with hearing aids applying the American Academy of Audiology Pediatric Amplification Guideline: Current practice and outcomes". Journal of the American Academy of Audiology 27.3 (2016): 204-218.

19. Rance G and Barker EJ. "Speech perception in children with auditory neuropathy/dyssynchrony managed with either hearing aids or cochlear implants". Otology and Neurotology 29.2 (2008): 179-182.

20. Eom JH., et al. "A Case of Auditory Neuropathy with Recovery of Normal Hearing". Korean Journal of Audiology 17.3 (2013): 138.

21. Psarommatis I., et al. "Transient infantile auditory neuropathy and its clinical implications". International Journal of Pediatric Otorhinolaryngology 70.9 (2006): 1629-1637.
22. Ganguly K and Poo M-m. "Activity-dependent neural plasticity from bench to bedside". Neuron 80.3 (2013): 729-741.

23. Scheibel RS., et al. "Effects of severity of traumatic brain injury and brain reserve on cognitive-control related brain activation". Journal of Neurotrauma 26.9 (2009): 1447-1461.

24. Beattie E Stellwagen., et al. "Control of synaptic strength by glial TNFalpha”. Science 295.5563 (2002): 2282-2285.

25. Becker JT., et al. "Pitt Brain Exercise and Training Program Aims to Improve Mind and Body Wellness in Individuals with Mild Cognitive Impairment Pittsburgh (2016).

26. Burda JE., et al. "Astrocyte roles in traumatic brain injury". Experimental Neurology 275 (2016): 305-315.

\section{Volume 3 Issue 6 June 2021 \\ (C) All rights are reserved by Suman $S$ Penwal and Chandrahas U Chandanshive.}

